\title{
ON A CLASS OF GENERALIZED FUNCTIONS
}

\author{
by K. ROWLANDS
}

(Received 4 November, 1974)

1. Introduction. Let $C_{0}^{\infty}(J)$ be the complex linear space of all infinitely differentiable functions $\varphi$ on the interval $J=(a, b)(-\infty \leqq a<0<b \leqq+\infty)$ such that $\varphi^{(k)}(0)=0$ for all non-negative integers $k$. Krabbe ([2], [3]) has defined a class of generalized functions on $J$ as an algebra $\mathscr{H}$ of linear operators on $C_{0}^{\infty}(J)$ and has developed an operational calculus for these operators†. Shultz ([6], Theorem 2.18) has recently shown that $\mathscr{M}$ is isomorphic to $\mathscr{D}_{+}^{\prime} \times \mathscr{D}_{-}^{\prime}$, where $\mathscr{D}_{+}^{\prime}$ (resp. $\mathscr{D}_{-}^{\prime}$ ) is the set of all distributions on $J$ whose supports are contained in $J_{+}=[0, b)\left(J_{-}=(a, 0]\right)$. In this paper we combine some of the ideas developed in [4] with results established in an earlier paper by Shultz to give an easier proof of the above result. Our methods also give a more direct proof of the main result (Theorem 1.22) of [2].

In the sequel, unless there is ambiguity, we shall denote $C_{0}^{\infty}(J)$ simply by $C_{0}^{\infty}$.

2. The algebra of generalized functions. We give $C_{0}^{\infty}$ the topology of uniform convergence for all derivatives on compact subsets of $J$; this topology is defined by the semi-norms

$$
r_{m, n}(\varphi)=\max _{k=0, \ldots, m}\left(\max _{a_{n} \leqq u \leqq b_{n}}\left|\varphi^{(k)}(u)\right|\right) \quad(m=0,1, \ldots ; n=1,2, \ldots),
$$

where

$$
a_{n}=\left\{\begin{array}{ll}
a(1-1 / n) & \text { if } a>-\infty \\
-n & \text { if } a=-\infty,
\end{array} \text { and } \quad b_{n}= \begin{cases}b(1-1 / n) & \text { if } b<+\infty \\
n & \text { if } b=+\infty\end{cases}\right.
$$

In this way $C_{0}^{\infty}$ becomes a Fréchet space. In the sequel, the topology defined by the seminorms $\left\{r_{m, n}\right\}$ will be referred to as the $C^{\infty}$ topology on $C_{0}^{\infty}$.

In ([2], [3]), Krabbe has defined the product of $\varphi, \psi \in C_{0}^{\infty}$ to be the function $\varphi \wedge \psi$ given by

$$
(\varphi \wedge \psi)(t)=\int_{0}^{t} \varphi(t-u) \psi(u) d u \quad(t \in J)
$$

For each $\varphi$ in $C_{0}^{\infty}$, define $\varphi_{+}$(resp. $\varphi_{-}$) to be the function which coincides with $\varphi$ on $J_{+}\left(J_{-}\right)$ and vanishes on $J_{-}\left(J_{+}\right)$, and let $\left(C_{0}^{\infty}\right)_{+}\left(\left(C_{0}^{\infty}\right)_{-}\right)$be the set of all such functions. Then $C_{0}^{\infty}=\left(C_{0}^{\infty}\right)_{+} \oplus\left(C_{0}^{\infty}\right)_{\text {. }}$. Also, it is easy to see that

$$
\varphi \wedge \psi=\varphi_{+} * \psi_{+}-\varphi_{-} * \psi_{-},
$$

where * denotes the convolution product, so that properties of the multiplicative operation $\wedge$ may be deduced from the corresponding properties of the convolution product. Thus, in view of the relationship between the operations $\wedge$ and * given by (2), there seems to be no advantage in using both symbols, and so in the sequel we shall denote the product $\varphi \wedge \psi$ by $\varphi * \psi$.

$\dagger$ It should be mentioned that Krabbe's theory of generalized functions has some resemblances to an earlier algebraic theory of generalized functions due to Weston [9]. 
It is straightforward to prove that, for any non-negative integer $k$,

$$
(\varphi * \dot{\psi})^{(k)}(t)=\left(\varphi * \dot{\psi}^{(k)}\right)(t) \quad(t \in J) .
$$

With multiplication of functions defined according to (1), $C_{0}^{\infty}$ becomes a linear, associative and commutative algebra; in fact, $C_{0}^{\infty}$ is an ideal in the algebra $L_{1}^{\text {loc }}$ of all complex-valued functions on $J$ which are Lebesgue-integrable over every compact subinterval of $J$, Multiplication is jointly continuous in $C_{0}^{\infty}$; this follows immediately from the inequality

$$
r_{m, n}(\varphi * \psi) \leqq\left(b_{n}+\left|a_{n}\right|\right) r_{0, n}(\varphi) r_{m, n}(\psi) .
$$

We define a sequence $\left\{\varphi_{n}\right\}$ of odd functions in $C_{0}^{\infty}(-\infty, \infty)$ by the formula

$$
\varphi_{n}(t)= \begin{cases}\frac{1}{\sqrt{\left(4 \pi n t^{3}\right)}} e^{-1 / 4 n t} & (t>0), \\ 0 & (t=0),\end{cases}
$$

and note the following properties of $\left\{\varphi_{n}\right\}$ :

(i) $\int_{0}^{\infty} \varphi_{n}(t) d t=1 \quad(n=1,2, \ldots)$;

(ii) for any $\delta>0, \lim _{n \rightarrow \infty} \int_{\delta}^{\infty} \varphi_{n}(t) d t=0$.

Let $\left\{\eta_{n}\right\}$ denote the restriction of $\varphi_{n}$ to $J$. Then we have the following

LEMmA 1. The sequence $\left\{\eta_{n}\right\}$ is an approximate identity for $C_{0}^{\infty}$.

Proof. We have to show that, for any $\psi$ in $C_{0}^{\infty}$,

$$
\psi=\lim _{n \rightarrow \infty} \psi * \eta_{n}
$$

By (2), to prove (4) it is sufficient to show that

and

$$
\psi_{+}=\lim _{n \rightarrow \infty} \psi_{+} *\left(\eta_{n}\right)_{+}
$$

$$
\psi_{-}=\lim _{n \rightarrow \infty} \psi_{-} *\left(\eta_{n}\right)_{-}
$$

We shall prove only (6); (5) may be proved using a similar method.

Let $\varepsilon>0, p$ be any positive integer, and $m$ any non-negative integer. Since $\psi^{(k)}(0)=0(k=0,1, \ldots)$, there exists a positive number $\delta_{k}^{\prime}$ such that $\left|\psi_{-}^{(k)}(v)\right|<\varepsilon$ whenever $-\delta_{k}^{\prime} \leqq v \leqq 0$. Let $\delta^{\prime}=\min \left(\delta_{0}^{\prime}, \ldots, \delta_{m}^{\prime}\right)$. By uniform continuity, there exists a positive number $\delta_{k}^{\prime \prime}$ such that

$$
\left|\psi_{-}^{(k)}(u-v)-\psi_{-}^{(k)}(u)\right|<\varepsilon
$$

whenever $a_{p} \leqq u \leqq 0$ and $-\delta_{k}^{\prime \prime} \leqq v \leqq 0$. Let $\delta^{\prime \prime}=\min \left(\delta_{0}^{\prime \prime}, \ldots, \delta_{m}^{\prime \prime}\right)$, and let $\delta=\min \left(\delta^{\prime}, \delta^{\prime \prime}\right)$. 
Now, since $\left(\eta_{n}\right)_{-}(t)=\varphi_{n}(t)$ for $t$ in $J_{-}$, we have

$$
r_{m, p}\left(\psi_{-} *\left(\eta_{n}\right)_{-}+\psi_{-}\right)=\max _{k=0, \ldots, m}\left(\max _{a_{p} \leqq u \leqq 0}\left|\int_{u}^{0} \psi_{-}^{(k)}(u-v) \varphi_{n}(v) d v+\psi_{-}^{(k)}(u)\right|\right) .
$$

If $-\delta \leqq u \leqq 0$, then

$$
\left|\int_{u}^{0} \psi_{-}^{(k)}(u-v) \varphi_{n}(v) d v+\psi_{-}^{(k)}(u)\right|<2 \varepsilon
$$

for $k=0, \ldots, m$. If $a_{p} \leqq u \leqq-\delta$, then

$$
\begin{gathered}
\left|\int_{u}^{0} \psi_{-}^{(k)}(u-v) \varphi_{n}(v) d v+\psi_{-}^{(k)}(u)\right|=\left|\int_{u}^{0} \psi_{-}^{(k)}(u-v) \varphi_{n}(v) d v-\int_{-\infty}^{0} \psi_{-}^{(k)}(u) \varphi_{n}(v) d v\right| \\
\leqq \int_{-\delta}^{0}\left|\psi_{-}^{(k)}(u-v)-\psi_{-}^{(k)}(u)\right|\left|\varphi_{n}(v)\right| d v+\int_{a_{p}}^{-\delta}\left|\psi_{-}^{(k)}(u-v) \| \varphi_{n}(v)\right| d v \\
+\int_{-\infty}^{-\delta}\left|\psi_{-}^{(k)}(u) \| \varphi_{n}(v)\right| d v .
\end{gathered}
$$

From (ii), it follows that there exists a positive integer $n_{0}$ such that, for $n \geqq n_{0}$,

$$
\int_{-\infty}^{-\delta}\left|\varphi_{n}(v)\right| d v<\varepsilon / r_{m, p}(\psi)
$$

Therefore, for $n \geqq n_{0}$ and $k=0, \ldots, m$, the right-hand side of (7) is less than $3 \varepsilon$. Hence, for $n \geqq n_{0}$,

$$
r_{m, p}\left(\psi_{-}+\psi_{-} *\left(\eta_{n}\right)_{-}\right)<3 \varepsilon
$$

and so, since $\varepsilon$ is arbitrary,

as required.

$$
\psi_{-}=\lim _{n \rightarrow \infty} \psi_{-} *\left(\eta_{n}\right)_{-}
$$

We note that, since $C_{0}^{\infty}$ has an approximate identity, $C_{0}^{\infty}$ has no non-zero annihilators.

In ([2], [3]), Krabbe has defined a generalized function on $J$ to be a multiplier on $C_{0}^{\infty} \dagger$; that is, a mapping $A$ of $C_{0}^{\infty}$ into itself such that

$$
A(\varphi * \psi)=A \varphi * \psi
$$

for all $\varphi, \psi$ in $C_{0}^{\infty}$. Let $\mathscr{M}$ denote the set of multipliers on $C_{0}^{\infty}$. It is well-known that the multipliers on a commutative algebra with no non-zero annihilators are linear, and that, if linear combinations and products of multipliers are defined in the usual way, then $\mathscr{M}$ is a commutative algebra $([8], \S 4)$. Also, every element of $\mathscr{M}$ is $C^{\infty}$ continuous ([1], Theorem 1 ).

It follows from (3) that the differential operator $D \in \mathscr{M}$. Also, each $f$ in $L_{1}^{\text {loc }}$ determines an element $F$ in $\mathscr{H}$ according to the equation

$$
F \varphi=f * \varphi
$$

† Krabbe did not use the term multiplier, but this seems now to be the standard terminology for this class of operators (see, for example, ([4], p. 51)). 
Krabbe ([2], (1.12)-(1.13)) has defined the operator of the function $f$ to be the operator $\{f(t)\}$ given by the equation

$$
\{f(t)\} \varphi=f * \varphi^{\prime}
$$

In our notation $\{f(t)\}$ is $D F$, and so, in particular, $\left\{\left(f_{1} * f_{2}\right)(t)\right\}$ is $D F_{1} F_{2}$. If $f, g \in L_{1}^{\text {loc }}$ and $F=G$, then it follows from a theorem of Titchmarsh ([7], p. 327, Theorem 152) that $f=g$ a.e. By the same argument we can show that $D F=D G$ implies that $f=g$ a.e.

We have now proved the following theorem, which is the main result of [2].

THEOREM 1 ([2], Theorem 1.22). The algebra $\mathscr{M}$ is commutative and each element of $\mathscr{H}$ is $C^{\infty}$ continuous. The operator $D \in \mathscr{M}$, and each $f$ in $L_{1}^{\text {loc }}$ determines an element $F$ of $\mathscr{H}$ according to (8). Also, for $f, g$ in $L_{1}^{\text {loc }}$, the following properties hold:

(a) $D\{(f * g)(t)\}=\{f(t)\}\{g(t)\}$;

(b) if $\{f(t)\}=\{g(t)\}$, then $f=g$ a.e.

For any $u>0$, the shift operator $I_{u}$ is the mapping of $C_{0}^{\infty}$ into itself defined by the equation

Then $I_{u} \in \mathscr{M}$ for all $u>0$.

$$
I_{u} \varphi(t)=\left\{\begin{array}{cl}
\varphi(t-u) & (u \leqq t<b) \\
0 & (-u<t<u) \\
\varphi(t+u) & (a<t \leqq-u)
\end{array}\right.
$$

Suppose $A \in \mathscr{M}$, and let $\varphi_{+}$be any element of $\left(C_{0}^{\infty}\right)_{+}$. By Lemma 1 ,

and so, since $A$ is $C^{\infty}$ continuous,

$$
\varphi_{+}=\lim _{n \rightarrow \infty} \varphi_{+} * \eta_{n},
$$

$$
A \varphi_{+}=\lim _{n \rightarrow \infty} \varphi_{+} * A \eta_{n}
$$

It follows that $A$ maps $\left(C_{0}^{\infty}\right)_{+}$into itself. Similarly, $A$ maps $\left(C_{0}^{\infty}\right)_{-}$into itself. Thus every element of $\mathscr{A}$ is linear, $C^{\infty}$ continuous, commutes with $I_{u}(u>0)$, and leaves invariant $\left(C_{0}^{\infty}\right)_{+}$ and $\left(C_{0}^{\infty}\right)_{\text {. }}$. Conversely, we have the following result.

THEOREM 2. Let $T$ be a $C^{\infty}$ continuous linear mapping of $C_{0}^{\infty}$ into itself with the following properties:

(a) $T I_{u}=I_{u} T$ for all $u>0$;

(b) $T$ leaves invariant $\left(C_{0}^{\infty}\right)_{+}$and $\left(C_{0}^{\infty}\right)_{-}+{ }^{\dagger}$

Then $T \in \mathscr{M}$.

Proof. Let $\varphi, \psi \in C_{0}^{\infty}$. Then

$$
\varphi * \psi=\varphi_{+} * \psi_{+}-\varphi_{-} * \psi_{-} .
$$

$\dagger$ There are $C^{\infty}$ continuous linear mappings of $C_{0}^{\infty}$ into itself which have property (a) but not (b); for example, the mapping $B$ given by

$$
B \varphi(t)=\varphi(-t) \quad\left(\varphi \in C_{0}^{\infty}, t \in J\right)
$$


Now $\varphi_{+} * \psi+$ may be approximated, in $C^{\infty}$ sense, by a function of the form

$$
\sum_{j=1}^{n} \alpha_{j} I_{u_{j}} \psi_{+},
$$

where $\alpha_{j}(j=1, \ldots, n)$ are complex-numbers (see the proof of $([4]$, Theorem 4)). Similarly, the function $\varphi_{-} * \psi_{-}$may be approximated, in the same sense, by a function of the form

$$
\sum_{j=1}^{m} \beta_{j} I_{w_{j}} \psi
$$

Hence $\varphi * \psi$ may be approximated by $\sum_{j=1}^{n} \alpha_{j} I_{u j} \psi_{+}-\sum_{j=1}^{m} \beta_{j} I_{w_{j}} \psi_{-}$. Since $T$ is linear,

$$
T \psi=T \psi_{+}+T \psi_{-},
$$

and, since $T$ has property (b), it follows that $T \psi_{+}=(T \psi)_{+}$and $T \psi_{-}=(T \psi)_{-}$. Consequently

$$
\varphi * T \psi=\varphi_{+} * T \psi_{+}-\varphi_{-} * T \psi_{-} .
$$

The function in (9) (resp. (10)) may be chosen in such a way that while $\varphi_{+} * \psi_{+}\left(\varphi_{-} * \psi_{-}\right)$can be approximated by (9) $((10)), \varphi_{+} * T \psi_{+}\left(\varphi_{-} * T \psi_{-}\right)$can be approximated by $\sum_{j=1}^{n} \alpha_{j} I_{u_{j}} T \psi_{+}$ $\left(\sum_{j=1}^{m} \beta_{j} I_{w j} T \psi_{-}\right)$. Hence $\varphi * T \psi$ may be approximated by the function

$$
\sum_{j=1}^{n} \alpha_{j} I_{n j} T \psi_{+}-\sum_{j=1}^{m} \beta_{j} I_{w_{j}} \mathrm{~T} \psi_{-\cdot}
$$

Since $T$ is linear and commutes with the shift operators, (11) is the same as

$$
T\left(\sum_{j=1}^{n} \alpha_{j} I_{w j} \psi_{+}-\sum_{j=1}^{m} \beta_{j} I_{w j} \psi_{-}\right)
$$

Finally, since $T$ is $C^{\infty}$ continuous, we have

as required.

$$
T(\varphi * \psi)=\varphi * T \psi,
$$

With convolution as multiplication, $\mathscr{D}_{+}^{\prime}$ (resp. $\left.\mathscr{D}_{-}^{\prime}\right)$ is a linear, associative, and commutative algebra. Let $\mathscr{H}_{+}\left(\mathscr{H}_{-}^{\prime}\right)$ denote the algebra of multipliers on $\left(C_{0}\right)_{+}^{\infty}\left(\left(C_{0}^{\infty}\right)_{-}\right)$. Shultz ([5], Theorem 4.3) has proved that $\mathscr{H}_{+}$is algebraically isomorphic to $\mathscr{D}_{+}^{\prime}$. Moreover, his proof may be easily modified to show that $\mathscr{H}_{-}$is isomorphic to $\mathscr{D}_{-}^{\prime}$. We may state Shultz's result as follows.

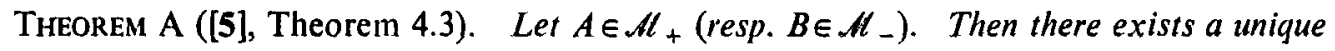
$\Phi \in \mathscr{D}_{+}^{\prime}\left(\Psi \in \mathscr{D}_{-}^{\prime}\right)$ such that

$$
A \varphi=\Phi * \varphi \quad(B \psi=\Psi * \psi)
$$

for all $\varphi \in\left(C_{0}^{\infty}\right)_{+}\left(\Psi \in\left(C_{0}^{\infty}\right)_{-}\right)$. Conversely, each $\Phi \in \mathscr{D}_{+}^{\prime}\left(\Psi \in \mathscr{D}_{-}^{\prime}\right)$ determines an element $A$ of $\mathscr{A}_{+}\left(B\right.$ of $\left.\mathscr{A}_{-}\right)$according to the above equation. The correspondence $A \leftrightarrow \Phi(B \leftrightarrow \Psi)$ is an isomorphism of $\mathscr{A}_{+}$onto $\mathscr{D}_{+}^{\prime}\left(\mathscr{H}_{-}\right.$onto $\left.\mathscr{D}_{-}^{\prime}\right)$. 
(In this connexion it is of interest to note that the above result of Shultz enables us to answer a question raised in ([4], p. 53), as follows. With the notation and terminology of [4], we have shown that the $C^{\infty}$ continuous $V$-operators on $C_{0}^{\infty}[0, \infty)$ are precisely the multipliers on $C_{0}^{\infty}[0, \infty)$, and so, if $T$ is a $C^{\infty}$ continuous $V$-operator on $C_{0}^{\infty}[0, \infty)$, then, by Theorem $A$, there exists a $\Phi \in \mathscr{D}_{+}^{\prime}$ such that

$$
T \varphi=\Phi * \varphi\left(\varphi \in C_{0}^{\infty}[0, \infty)\right)
$$

It follows that $T$ satisfies $\left(P_{2}\right)$, and so there are no $C^{\infty}$ continuous $V$-operators on $C_{0}^{\infty}[0, \infty)$ which do not have property $\left(P_{2}\right)$.)

We note that, if linear combinations of elements of $\mathscr{D}_{+}^{\prime} \times \mathscr{D}_{-}^{\prime}$ are defined in the usual way and multiplication is defined according to the equation

$$
\left(\Phi_{1}, \Psi_{1}\right) \otimes\left(\Phi_{2}, \Psi_{2}\right)=\left(\Phi_{1} * \Phi_{2}, \Psi_{1} * \Psi_{2}\right),
$$

then $\mathscr{D}_{+}^{\prime} \times \mathscr{D}_{-}^{\prime}$ becomes a linear, associative, and commutative algebra.

Finally, we give an easier proof of ([6], Theorem 2.18).

THEOREM 3. The correspondence $T \leftrightarrow(\Phi, \Psi)\left(T \in \mathscr{M},(\Phi, \Psi) \in \mathscr{D}_{+}^{\prime} \times \mathscr{D}_{-}^{\prime}\right)$, where

$$
T \varphi=\Phi * \varphi_{+}+\Psi * \varphi_{-}\left(\varphi \in C_{0}^{\infty}\right),
$$

is an isomorphism of $\mathscr{M}$ onto $\mathscr{D}_{+}^{\prime} \times \mathscr{D}_{-}^{\prime}$.

Proof. Suppose that $T \in \mathscr{M}$, and let $T_{+}$(resp. $T_{-}$) denote the restriction of $T$ to $\left(C_{0}^{\infty}\right)_{+}$ $\left(\left(C_{0}^{\infty}\right)_{-}\right)$. Then, since $T_{+}\left(T_{-}\right)$leaves invariant $\left(C_{0}^{\infty}\right)_{+}\left(\left(C_{0}^{\infty}\right)_{-}\right), T_{+} \in \mathscr{M}_{+}\left(T_{-} \in \mathscr{M}_{-}\right)$and

$$
T \varphi=T_{+} \varphi_{+}+T_{-} \varphi_{-} .
$$

By Theorem $\mathrm{A}$, there exists a unique $(\Phi, \Psi)$ in $\mathscr{D}_{+}^{\prime} \times \mathscr{D}_{-}^{\prime}$ such that

$$
T \varphi=\Phi * \varphi_{+}+\Psi * \varphi_{-} .
$$

Conversely, each $(\Phi, \Psi)$ in $\mathscr{D}_{+}^{\prime} \times \mathscr{D}_{-}^{\prime}$ determines an element $T$ of $\mathscr{M}$ according to (12). It is straightforward to show that the correspondence $T \leftrightarrow(\Phi, \Psi)$ is an isomorphism of the algebra $\mathscr{M}$ onto the algebra $\mathscr{D}_{+}^{\prime} \times \mathscr{D}_{-}^{\prime}$.

\section{REFERENCES}

1. T. K. Boehme, Continuity and perfect operators, J. London Math. Soc. 39 (1964), 355-358.

2. G. Krabbe, Linear operators and operational calculus, Part I, Studia Mathematica 40 (1971), 199-223.

3. G. Krabbe, An algebra of generalized functions on an open interval: two-sided operational calculus, Pacific J. Math. 42 (1972), 381-411.

4. K. Rowlands, A convolution-integral representation for a class of linear operators, Proc. Cambridge Phil. Soc. 71 (1972), 51-60. $1-25$.

5. H. Shultz, Linear operators and operational calculus, Part II, Studia Mathematica 41 (1972),

6. H. Shultz, An algebra of distributions on an open interval, Trans. American Math. Soc. 169 (1972), 163-181. 
7. E. C. Titchmarsh, Theory of Fourier integrals (Oxford, 1937).

8. J. D. Weston, Positive perfect operators, Proc. London Math. Soc. (3) 10 (1960), 545-565.

9. J. D. Weston, An algebraic theory of generalized functions, Nieuw Archief voor Wiskunde (3) 18 (1970), 211-249.

Department of Pure Mathematics

UNiversity COLlege of Wales

ABERYSTWYTH 